\title{
Is it possible to eliminate sternal wound infection after cardiac surgery?
}

\author{
Shahzad G. Raja, MRCS, FRCS(C-Th) \\ Department of Cardiac Surgery, Harefield Hospital, London, United Kingdom
}

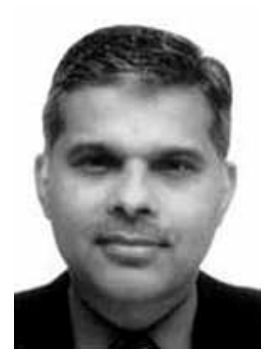

Kardiochirurgia i Torakochirurgia Polska 2014; 11 (2): 230-232

\begin{abstract}
„If you always do what you've always done, You'll always get what you've always got."

Henry Ford (1863-1947)
\end{abstract}

Sternal wound infection (SWI) is a serious and expensive complication after cardiac surgical procedures. A large array of risk factors including obesity, chronic obstructive pulmonary disease, diabetes, reoperation, use of bilateral internal thoracic artery (ITA) conduits, long operation time, low cardiac output, prolonged ventilation, and re-exploration for bleeding are associated with its occurrence [1]. At the same time a variety of therapeutic options such as debridement with early or delayed closure, closed continuous irrigation, vacuum-assisted closure, partial or complete sternectomy with flap reconstruction and omental transposition [1], or the innovative but expensive titanium rib bridge system (STRATOS) [2] are available for dealing with this frustrating and often lethal complication after routine cardiac surgery.

Despite our enormous understanding of the pathophysiology of SWI after cardiac surgery and massive advances in its management it remains a major physical disability, psychological impediment, as well as a financial liability.

At Harefield Hospital, over the past five years, we have shifted our focus from management of SWI to its pre-emption and prevention. This paradigm shift has resulted in an enormous reduction in the rates of SWI infection at our institution (Fig. 1), encouraging everyone to believe that it is possible to eliminate SWI after cardiac surgery. We have adopted a three-pronged strategy to tackle SWI. This strategy involves focus on modifiable pre-, intra- and postoperative risk factors, adoption of a standardized sternal wound care bundle, and an efficient infection control, prevention and surveillance service.

As a first step we risk stratify all our cardiac surgical patients on admission using the Brompton \& Harefield Infection Score (BHIS). This risk scoring system categorizes patients as low (BHIS $=0-1$ ), medium (BHIS $=2-3$ ), or high risk $(\mathrm{BHIS} \geq 4)$ for SWI taking into consideration whether they are female (score $=2$ ), diabetic (score $=1$ ), $\mathrm{Hb}_{1 \mathrm{Ac}}>7.5 \%($ score $=3), \mathrm{BMI} \geq 35$ (score $=2$ ), ejection fraction $\leq 45 \%($ score $=1)$, and emergency surgery (score $=2$ ). Identification of patients who are at high or medium risk for SWI then triggers a cascade of events and interventions aimed at eliminating the occurrence of SWI. One of the key areas we have aggressively focused on is the achievement of normoglycaemia in all diabetics, both pre- as well as postoperatively. There is evidence to suggest that better control of pre- and postoperative blood glucose levels in patients with diabetes lowers the incidence of sternal wound complications, particularly deep SWI after cardiac surgery [3]. Our commitment to achieving this target is reflected by the fact that we have developed a dedicated diabetic specialist nurse service with input from a consultant endocrinologist.

We have recently described our standardized sternotomy wound care bundle in detail [4]. We clip all the side branches during ITA harvest and use the point haemostasis technique to minimize leaving charred tissue on the inside of the chest wall, which could potentially act as a culture medium for microbes. We routinely wash the chest cavity at the end of the procedure with at least a litre of warm saline to remove all debris and use a double wire technique to close the sternum. Our aforementioned sternal wiring technique exerts force both from top to bottom and across the midline, thus achieving a secure closure and fixing any fractures at the same time. We firmly believe that deep wound infection occurrence might be a consequence of technical pitfalls, predominant amongst them being suboptimal sternal wiring technique [5]. Again we standardized this wiring technique in 2007 and it could be a contributory factor to the decline in sternal wound complications in our experience. At about the same time we started using a gentamicin-collagen sponge (Collatamp ${ }^{\circledR}$, EUSA Pharma [Europe], Oxford, United Kingdom) for all high-risk patients including diabetics with BIMA harvest. Prior to closure of the sternum and after placement of the sternal wires, a single Collatamp ${ }^{\circledR}$ sponge $\left(20 \times 5 \times 0.5 \mathrm{~cm} ; 1 \mathrm{~cm}^{2}\right.$ containing $2.8 \mathrm{mg}$

Address for correspondence: Shahzad G. Raja BSc, MBBS, MRCS, FRCS(C-Th), Department of Cardiac Surgery, Harefield Hospital, Hill End Road, Harefield, UB9 6JH, London, United Kingdom. tel. +441895828550, fax +441895828992, e-mail: drrajashahzad@hotmail.com 
CABG SSI rate 2003-2012

All categories (superficial/deep/organ or space) detected admission or re-admission

Sternal and donor SSI

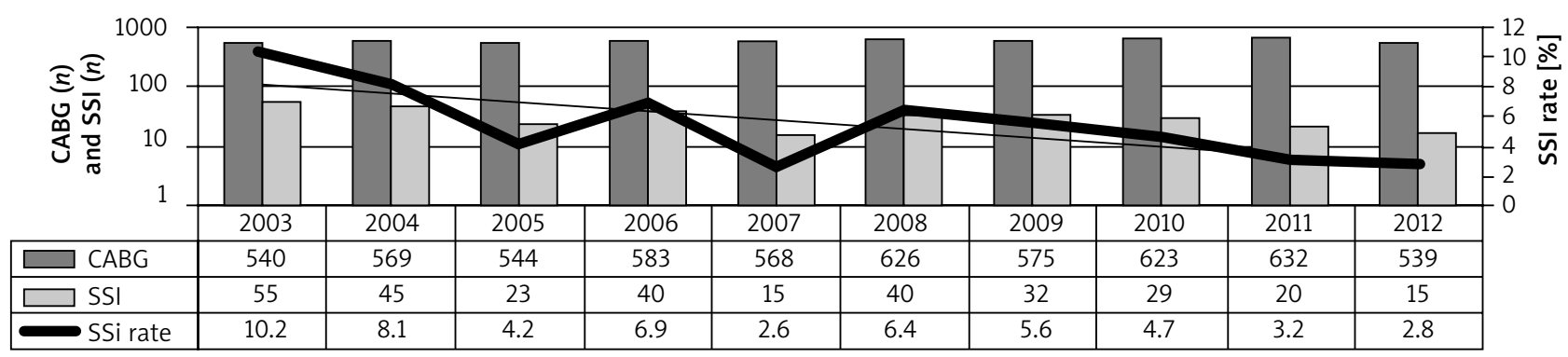

Incidences of surgical site infection, all categories (superficial/deep/organ)

CABG SSI detected during in patient stay (primary/re-admit)

HH April 2012 - March 2013

12 month average $=2.7$ per 100 operations 08/05/2013 surveillance data

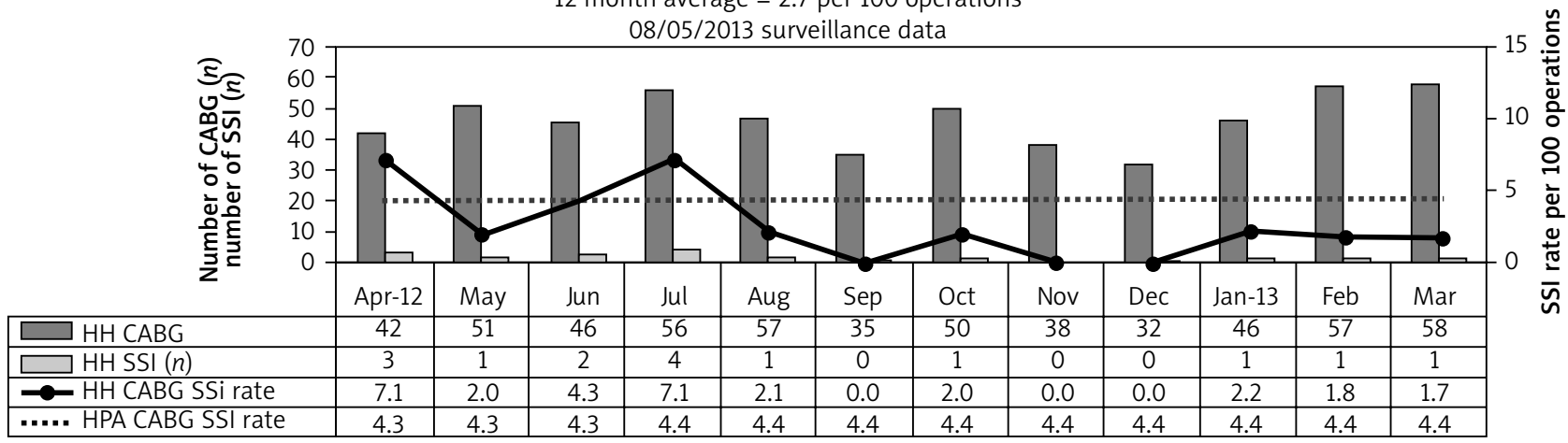

Fig. 1. Yearly trends in the rate of sternal wound infection at Harefield Hospital

of native collagen fibrils of equine origin and also containing $2 \mathrm{mg}$ of gentamicin sulphate, equivalent to 1.10-1.43 $\mathrm{mg}$ of gentamicin) is implanted retrosternally without premoistening in all cases deemed high risk for sternal wound complications. Sternal wires are then tightened. There is evidence from a number of randomized controlled trials to suggest that Collatamp ${ }^{\circledR}$ reduces the incidence of sternal wound infection [6] and our experience supports the findings of these trials [7]. In addition to these intraoperative measures, we keep all our surgical wounds (sternotomy and vein harvest site) covered with a waterproof, bacteriaproof dressing with a see-through absorbent pad (OpSite Post-op Visible ${ }^{\circledR}$, Smith \& Nephew, UK) for a period of 5-7 days postoperatively. This dressing with greater visibility and superior absorbency allows us to monitor progress of the wound as often as we like without unnecessary dressing changes. This reduces disturbance to the wound bed, minimizes the risk of infection and helps to provide a costeffective solution. It also allows the patient to shower with the dressing in situ without unnecessary dressing changes. Our rationale for keeping the wound covered for 5-7 days is that the majority of the wounds are infected by crosscontamination in the immediate postoperative period and keeping them covered during this period minimizes the risk significantly. Other measures which have contributed significantly to reducing SWI include adoption of endoscopic vein harvesting as a default strategy for all coronary artery bypass grafting patients requiring vein grafts and manda- tory wearing of a supportive bra for at least 4 weeks when breast cup $\geq \mathrm{D}$.

Finally, we realized after battling with the menace of SWI for years that a mechanism must be in place at institutional level that co-ordinates efforts intra-institutionally and collaborates with external agencies to enable us to achieve the difficult but possible task of elimination of SWI after cardiac surgery. To achieve this target we developed an infection prevention, control, and surveillance service run by specialist nurses with input from cardiac surgeons as well as microbiologists. This service not only ensures that efforts to eliminate SWI are well coordinated but also fulfils the extremely useful task of providing root cause analysis for every case of SWI, thus enabling us to constantly review and renew our resolve to abolish SWI.

We are finally beginning to reap the rewards of our labour. We have reduced our rates of all SWI to less than $2 \%$ and eliminated mediastinitis from our practice. This has all been possible because we decided to do something different!

\section{References}

1. Tang GH, Maganti M, Weisel RD, Borger MA. Prevention and management of deep sternal wound infection. Semin Thorac Cardiovasc Surg 2004; 16 62-69.

2. Kim WK, Kim JB, Kim GS, Jung SH, Choo SJ, Chung CH, Lee JW. Titanium plate fixation for sternal dehiscence in major cardiac surgery. Korean J Thorac Cardiovasc Surg 2013; 46: 279-284. 
3. Schmeltz LR, DeSantis AJ, Thiyagarajan V, Schmidt K, O'Shea-Mahler E, Johnson D, Henske J, McCarthy PM, Gleason TG, McGee EC, Molitch ME. Reduction of surgical mortality and morbidity in diabetic patients undergoing cardiac surgery with a combined intravenous and subcutaneous insulin glucose management strategy. Diabetes Care 2007; 30: 823-828.

4. Raja SG, Salhiyyah K, Rafiq MU, Navaratnarajah M, Chudasama D, Walker CP, Amin F, Amrani M. In-hospital outcomes of pedicled bilateral internal mammary artery use in diabetic and nondiabetic patients undergoing off-pump coronary artery bypass grafting: single-surgeon, single-center experience. Heart Surg Forum 2013; 16: E1-7.
5. Bottio T, Rizzoli G, Vida V, Casarotto D, Gerosa G. Double crisscross sterna wiring and chest wound infections: a prospective randomized study. J Thorac Cardiovasc Surg 2003; 126: 1352-1356.

6. Raja SG. Local application of gentamicin-containing collagen implant in the prophylaxis and treatment of surgical site infection following cardiac surgery. Int J Surg 2012; 10 Suppl 1: S10-4.

7. Raja SG, Salhiyyah K, Rafiq MU, Felderhof J, Amrani M. Impact of gentamicin-collagen sponge (Collatamp) on the incidence of sternal wound infection in high-risk cardiac surgery patients: a propensity score analysis. Heart Surg Forum 2012; 15: E257-261. 